\title{
Modelagem da mortalidade em povoamentos de Pinus taeda L.
}

\author{
Modelling mortality in Pinus taeda L. stands
}

\author{
Rodrigo Otávio Veiga de Mirandaㄹ, Afonso Figueiredo Filho², Sebastião do Amaral Machado ${ }^{3}$, \\ Renato Vinícius Oliveira Castro ${ }^{4}$, Luan Demarco Fiorentin ${ }^{5}$ e Luiz Gastão Bernett ${ }^{6}$
}

\begin{abstract}
Resumo
A mortalidade de árvores é um dos fatores com influência direta no crescimento e produção florestal. Sua avaliação requer atenção pois representa um componente essencial na simulação da dinâmica florestal. O objetivo deste trabalho foi ajustar e avaliar modelos matemáticos para estimar a probabilidade de mortalidade de povoamentos de Pinus taeda L. Os dados foram provenientes de parcelas permanentes em povoamentos não desbastados, localizados na região de Telêmaco Borba, estado do Paraná, com idade de 3,2 a 21 anos. Inicialmente, foi avaliada a dinâmica da mortalidade, por idade e classe de sítio. 0 comportamento do número de árvores vivas e mortas por classe diamétrica também foi analisado, assim como determinada a amplitude diamétrica de ocorrência da mortalidade. Foram testados quatro modelos de probabilidade de mortalidade por classe diamétrica. A ocorrência de mortalidade foi observada a partir dos cinco anos de idade, até a classe diamétrica de $28 \mathrm{~cm}$ e entre o diâmetro mínimo até $75 \%$ do diâmetro médio aritmético da parcela. $O$ modelo exponencial estimou melhor o número de árvores mortas por hectare, para as três classes de sítio, o qual empregou o índice de área basal (BAl) como variável independente, assim como o sítio $(\mathrm{S})$, centro da classe diamétrica $\left(\mathrm{CC}_{\mathrm{d}}\right)$, idade $(\mathrm{I})$ e área basal da classe diamétrica $\left(\mathrm{G}_{\text {classe }}\right)$.
\end{abstract}

Palavras-chave: Mortalidade regular; probabilidade de mortalidade; função exponencial.

\begin{abstract}
Tree mortality is a factor with direct influence on forest growth and production. Its evaluation requires attention in order to represent an essential component in the simulation of forest dynamics. The aim of this study was to fit and evaluate mathematical models to estimate the probability of tree mortality in Pinus taeda L. stands. Data were obtained from permanent non-thinned plots, located in the region of Telemaco Borba, State of Parana, Brazil, aged from 3.2 to 21 years. Initially mortality dynamics was evaluated by age and site class. Also, the behavior of the number of living trees and mortality were analyzed by diameter class, as well as specifying the diametric range of mortality. The mortality of the stands was estimated using four probability of mortality models by diameter class. The occurrence of mortality was observed from five years of age until the diametric class of $28 \mathrm{~cm}$ and the minimum diameter until $75 \%$ of the arithmetical mean diameter of the plot. The exponential model that estimated the number of dead trees per hectare for the three site classes, used the basal area index (BAl) as independent variable, as well as site (S), center of diameter class $\left(\mathrm{CC}_{\mathrm{d}}\right)$, age $(\mathrm{I})$ and basal area by diameter class $\left(\mathrm{G}_{\text {classe }}\right)$.
\end{abstract}

Keywords: Regular mortality; probability of mortality; exponential function.

\footnotetext{
1Doutor em Engenharia Florestal. UFPR - Universidade Federal do Paraná. Rua Lothário Meissner, 632 - Jardim Botânico - 80210-170 - Curitiba, PR, Brasil. E-mail: rov_miranda@yahoo.com.br.

2Professor Associado do Departamento de Engenharia Florestal. UNICENTRO - Universidade Estadual do Centro-Oeste / Centro de Ciências Agrárias e Ambientais. PR-153 - KM 7 - Bairro Riozinho - Caixa-postal: 121 - 84500-000 - Irati, PR , Brasil. E-mail: afigfilho@gmail.com.

3Professor Titular do Departamento de Ciências Florestais. UFPR - Universidade Federal do Paraná. Rua Lothário Meissner, 632 - Jardim Botânico - 80210-170 - Curitiba, PR, Brasil. E-mail: profsamachado@gmail.com.

${ }^{4}$ Professor Adjunto no Departamento de Ciências Agrárias. UFSJ - Universidade Federal de São João Del Rei / CSL - Campus Sete Lagoas. Rua Sétimo Moreira Martins - Itapoã II - 35702-031 - Sete Lagoas, MG, Brasil. E-mail: renatocastro@ufsj.edu.br.

${ }^{5}$ Doutorando em Engenharia Florestal. UFPR - Universidade Federal do Paraná. Rua Lothário Meissner, 632 - Jardim Botânico - 80210-170 - Curitiba, PR, Brasil. E-mail: luanfiorentin@ hotmail.com. 6Engenheiro Florestal. Klabin S.A. Fazenda Monte Alegre s/n - Harmonia - 84275-000 - Telêmaco Borba PR, Brasil. E-mail: gastao@klabin.com.br.
} 
Miranda et al. - Modelagem da mortalidade em povoamentos de Pinus taeda L.

\section{INTRODUÇÃO}

Um dos fatores que atuam na dinâmica da floresta é a mortalidade de árvores e, assim, sua avaliação deve ser muito criteriosa (YANG et al., 2003). Em povoamentos equiâneos, geralmente ocorrem dois tipos de mortalidade, a regular e a irregular (WEISKITTEL et al., 2011). A primeira se refere àquela provocada pela competição, idade, fatores genéticos ou senescência (DOBBERTIN; BIGING, 1998; MIRANDA et al., 1989). Em contrapartida, a mortalidade irregular é comumente associada a algum evento catastrófico, como quedas de árvores provocadas por ventos, fogo, inseto, doenças e, em povoamentos de Pinus, ainda é comum o ataque do macaco-prego (Sapajus nigritus, Goldfuss, 1809), podendo gerar danos significativos (LIEBSCH et al., 2015).

Em sistemas de predição do crescimento e produção florestal, um componente essencial é o emprego de modelos para predição da probabilidade de mortalidade de árvores individuais (BUFORD; HAFLEY, 1985; CAO, 1997). Associando esses modelos àqueles de crescimento em diâmetro e altura de árvores individuais, pode-se prever o desenvolvimento do povoamento florestal ao longo do tempo (TECK; HILT, 1990). A precisão das estimativas de crescimento está relacionada com a precisão da estimativa do número de árvores vivas (ou mortas) (SIMS et al., 2009).

A mortalidade de árvores pode ser estimada a partir de uma função baseada no estado fisiológico da árvore; por meio da estimativa de alguma condição fisiológica da árvore; e pela determinação da probabilidade da mortalidade da árvore individual dentro de um grupo com características semelhantes (GLOVER; HOOL, 1979). Recentemente, grande parte dos modelos de sobrevivência (ou mortalidade) foi desenvolvida para a árvore individual (MABVURIRA; MIINA, 2002), sendo comum o uso de modelos estatísticos.

O modelo de regressão logística é geralmente aplicado para modelar a probabilidade de morte para a árvore individual (CASTEL-BRANCO, 2014). A propósito, quase todas as equações de mortalidade em nível de árvore usam a regressão logística (WEISKITTEL et al., 2011), uma vez que proporciona bons resultados e pela facilidade de ajuste. Na literatura florestal também podem ser encontrados outros modelos para estimar a probabilidade de mortalidade, como pode ser visto nos trabalhos de Castro (2011) e Martins (2011).

Com isso, verifica-se a importância do conhecimento da mortalidade em povoamentos florestais, sobretudo no desenvolvimento de estratégias vinculadas ao manejo florestal. Diante do exposto, o objetivo deste trabalho foi ajustar e avaliar modelos para estimar a probabilidade de mortalidade de povoamentos de P. taeda situados na região dos Campos Gerais, estado do Paraná.

\section{MATERIAL E MÉTODOS}

\section{Descrição da Área}

De acordo com a classificação de Köppen, o clima do local pertence ao tipo Cfb. A temperatura média anual é de $23{ }^{\circ} \mathrm{C}$, com temperatura mínima e máxima histórica igual a $-5,2$ e $37{ }^{\circ} \mathrm{C}$, respectivamente. A precipitação anual média varia em torno de $1.470 \mathrm{~mm}$ ao ano. O relevo da região de estudo é suavemente ondulado e a altitude média de 850 m acima do nível do mar (FIGURA, 2010; NUNES et al., 2010).

\section{Descrição dos dados}

Os dados empregados nesta pesquisa provêm de povoamentos de Pinus taeda L. não desbastados, advindos de pomar clonal de sementes de primeira geração, com espaçamento inicial de plantio de 2,5 m × 2,5 m, situados na região de Telêmaco Borba, estado do Paraná.

As informações foram obtidas de parcelas permanentes, distribuídas com uma intensidade de, aproximadamente, uma parcela por hectare. As parcelas assumiram áreas diferentes, uma vez que sua alocação consistiu na contagem de 10 linhas de plantio e 10 plantas, sendo realizado o esquadrejamento dos limites. Em cada uma, foram medidos os diâmetros à altura do peito (DAP) (medido a 1,3 $\mathrm{m}$ do solo) de todas as árvores, altura total (h) das 10 primeiras e as alturas das árvores dominantes conforme metodologia proposta por Assmann (1970). No total, foram utilizados dados de 1.056 parcelas, medidas nos anos de 2011 a 2014, com idade variando de 3,2 a 21 anos, na qual $90 \%$ das parcelas possuiu apenas duas medições, 10\% com 3 medições, e apenas uma parcela medida nos quatro anos (2011 a 2014). 
Para a distinção das classes de sítio foi utilizado o modelo biológico de Chapman-Richards, cuja equação resultante foi $\mathrm{h}_{\text {dom }}=41,224399\left(1-\mathrm{e}^{-0,061832 \mathrm{I}}\right)^{1,156161}$, com erro padrão da estimativa percentual $\left(\mathrm{S}_{\mathrm{yx}}\right)$ igual a $9,5 \%$, em que $\mathrm{h}_{\mathrm{dom}}$ e I são a altura dominante $(\mathrm{m})$ e idade (anos), respectivamente. O método para a construção das curvas de índices de sítio foi o da curva-guia e a idade índice considerada foi de 18 anos. Três classes de sítio foram determinadas, com amplitude de variação de 3,9 m. A classe I compreendeu alturas dominantes na idade índice de 28,1 a 33,0 m; a segunda classe englobou alturas dominantes entre 23,1 a $28,0 \mathrm{~m}$; e a classe III envolveu alturas dominantes de 18,0 a 23,0 m, representando a classe de produtividade alta, média e baixa, respectivamente.

Para estimar as alturas totais das árvores, foi ajustado um modelo hipsométrico genérico dado pela equação $h=e^{0,72145+0,02395 S-5,90105 D \cdot A P^{-1}+0,726691 \mathrm{nI}}, \operatorname{com~S}$ yx igual a 6,7\%, em que h é a altura total (m), S o índice de sítio (m), DAP e I como já definidos.

Na Tabela 1 estão apresentados os valores mínimos, médios e máximos do número de árvores vivas por hectare, por classe de sítio e nas idades de 6 a 18 anos, em intervalos bianuais.

Tabela 1. Estatísticas descritivas para o número de árvores por hectare, por classe de sítio e idade.

Table 1. Descriptive statistics for number of trees per hectare by site and age class.

\begin{tabular}{|c|c|c|c|c|c|c|c|c|c|c|c|c|}
\hline \multirow{2}{*}{ Idade } & \multicolumn{4}{|c|}{ Sítio I $(30,5 \mathrm{~m})$} & \multicolumn{4}{|c|}{ Sítio II (25,5 m) } & \multicolumn{4}{|c|}{ Sítio III $(20,5 \mathrm{~m})$} \\
\hline & Min & Med & Max & CV\% & Min & Med & Max & CV\% & Min & Med & Max & CV\% \\
\hline 6 & 716 & 1.462 & 1.791 & 11,6 & 638 & 1.435 & 1.845 & 12,1 & 797 & 1.361 & 1.655 & 18,3 \\
\hline 8 & 730 & 1.461 & 1.619 & 14,6 & 732 & 1.340 & 1.687 & 13,9 & 816 & 1.214 & 1.508 & 17,7 \\
\hline 10 & 1.147 & 1.460 & 1.650 & 8,0 & 738 & 1.334 & 1.633 & 15,0 & 640 & 1.211 & 1.574 & 21,5 \\
\hline 12 & 773 & 1.380 & 1.617 & 11,7 & 893 & 1.323 & 1.600 & 13,2 & 920 & 1.123 & 1.558 & 17,7 \\
\hline 14 & 733 & 1.296 & 1.440 & 16,4 & 796 & 1.268 & 1.488 & 11,4 & 753 & 1.028 & 1.339 & 18,9 \\
\hline 16 & 1.003 & 1.255 & 1.434 & 9,6 & 947 & 1.253 & 1.712 & 11,7 & 916 & 1.019 & 1.123 & 14,4 \\
\hline 18 & 1.154 & 1.202 & 1.239 & 3,2 & 912 & 1.203 & 1.394 & 9,8 & 1.001 & 1.001 & 1.001 & - \\
\hline
\end{tabular}

Em que: $\mathrm{CV} \%$ = coeficiente de variação; Min, Med e Max = valores mínimos, médios e máximos, respectivamente.

\section{Análise da mortalidade do povoamento}

O total de árvores mortas por hectare foi avaliado por classe de sítio e idade. Ainda, foi observado o comportamento do número de árvores vivas e mortas por classe diamétrica, nas idades de 5, 10, 15 e 20 anos, considerando o povoamento sem distinção em classes de sítio. A amplitude diamétrica adotada foi de $2 \mathrm{~cm}$, visto que se trata de uma amplitude bastante difundida em empresas florestais.

\section{Modelos de mortalidade}

A mortalidade por hectare foi estimada por meio da probabilidade de mortalidade por classe diamétrica, na qual foram testados quatro modelos não lineares (Tabela 2). Esses modelos foram selecionados conforme resultados dispostos na literatura florestal (MARTINS et al., 2011; SIMS et al., 2009; THAPA, 2014).

Tabela 2. Modelos testados para estimar a probabilidade de mortalidade.

Table 2. Tested models for estimating the probability of mortality.

\begin{tabular}{|c|c|c|}
\hline $\mathbf{N}^{0}$. & Modelo & Especificação \\
\hline 1 & Logístico (a) & $\operatorname{Pm}=\left(1+e^{\beta_{0}+\beta_{1} I C_{i}}\right)^{-1}+\varepsilon_{i}$ \\
\hline 2 & Logístico (b) & $P m=\left(1+e^{\beta_{0}+\beta_{1} I C_{1}+\beta_{2} C C_{d}+\beta_{3} h_{\text {media }}+\beta_{4} I+\beta_{3} G_{\text {clusse }}+\beta_{0} S}\right)^{-1}+\varepsilon_{i}$ \\
\hline 3 & Exponencial & $\mathrm{Pm}=\beta_{0}+\mathrm{e}^{\left(\beta_{1}+\beta_{2} \mathrm{IC}+\beta_{3} \mathrm{CC}_{\mathrm{d}}+\beta_{4} \mathrm{~h}_{\text {medu }}+\beta_{3} \mathrm{I}+\beta_{6} \mathrm{G}_{\text {clusse }}+\beta_{\mathrm{S}} \mathrm{S}\right)}+\varepsilon_{\mathrm{i}}$ \\
\hline 4 & Buchman & 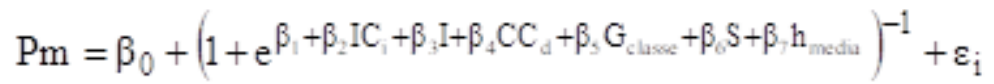 \\
\hline
\end{tabular}

Em que: $P_{m}=$ probabilidade de mortalidade por classe diamétrica; $I C=$ índice de competição; $C_{d}=$ centro da classe diamétrica (cm); $h_{m e d}=$ altura total média da classe diamétrica $(\mathrm{m}) ; \mathrm{I}=$ idade da parcela (anos); $\mathrm{G}_{\text {classe }}=$ área basal da classe diamétrica $\left(\mathrm{m}^{2}\right.$. ha- $\mathrm{I}$. classe); $\mathrm{S}=$ índice de sítio $(\mathrm{m}) ; \beta_{i}=$ coeficientes a serem estimados; $\varepsilon i=$ erro aleatório da equação. 
O modelo logístico foi utilizado de duas maneiras. Na primeira, a probabilidade de mortalidade foi estimada somente em função do índice de competição (CASTRO, 2011; MARTINS, 2011). No segundo caso, além do índice de competição, foi estimada em função de variáveis do povoamento (SIMS et al., 2009, THAPA, 2014), assim como o modelo exponencial (MARTINS, 2011; PAYANDEH, 1983). Finalmente, o modelo 4 foi citado em Buchman, et al. (1983), porém, com modificações, uma vez que foram incluídas outras variáveis.

A probabilidade de mortalidade foi calculada com a expressão $\mathrm{Pm}_{\mathrm{i}}=\mathrm{N}_{\mathrm{m}}\left(\mathrm{N}_{\mathrm{v}}+\mathrm{N}_{\mathrm{m}}\right)^{-1}$, semelhante àquela empregada por Machado et al. (2002), Martins (2011) e Castro (2012), considerada de ocorrência anual (FLEWELLING; MONSERUD, 2002), em que $\mathrm{Pm}_{\mathrm{i}}$ é a probabilidade de mortalidade anual na classe diamétrica $\mathrm{i} ; \mathrm{N}_{\mathrm{v}}$ e $\mathrm{N}_{\mathrm{m}}$ representam o número de árvores vivas e mortas da parcela por hectare, respectivamente, para uma mesma idade.

Ao contabilizar o número de árvores vivas da parcela, em determinada idade, obteve-se o $\mathrm{N}_{\mathrm{v}}$. Para o cálculo de $\mathrm{N}_{\mathrm{m}^{\prime}}$ foi observado o ano correto em que a mortalidade ocorreu, para que essa variável não fosse computada repetidas vezes em sucessivas medições e, consequentemente, superestimada. A mortalidade só foi observada a partir dos 5 anos de idade. Dessa forma, na ocasião da primeira medição de alguma parcela, caso sua idade se situasse acima de 5 anos, o número de árvores mortas foi a média até a idade da parcela, iniciando-se aos 5 anos.

Três índices de competição independentes da distância foram calculados para serem testados como variável independente nos modelos, de modo que cada modelo foi ajustado três vezes, uma para cada índice de competição. Os índices testados foram os de Stage (1973), dados pela expressão $\mathrm{d}_{i}^{2} \mathrm{q}^{-2}$ (BAI - IC.1) e $\sum_{i=1}^{\mathrm{g}_{i}}$ (BAL - IC.2), e o índice de Tomé e Burkhart (1989), expresso por $\mathrm{d}_{i} \mathrm{~d}_{\max }^{-1}$ (IC.3). Em que $\mathrm{d}_{\mathrm{i}}{ }_{\mathrm{i}}^{=1}=\mathrm{DAP}$ da árvore-objeto $(\mathrm{cm}) ; \mathrm{q}=$ diâmetro quadrático das árvores da unidade amostral $(\mathrm{cm}) ; \mathrm{g}_{\mathrm{i}}=$ área transversal das árvores maiores à árvore-objeto $\left(\mathrm{m}^{2}\right)$; e $\mathrm{d}_{\max }=\mathrm{DAP}$ máximo da unidade amostral $(\mathrm{cm})$.

\section{Avaliação dos modelos}

O método utilizado para ajustar os modelos foi o algoritmo de Levenberg-Marquardt. Tanto para a regressão como para os coeficientes, o nível de significância adotado foi de $5 \%$ ( $p$-valor $\leq 0,05$ ). Para avaliar a qualidade dos modelos ajustados, foi empregado o erro padrão da estimativa percentual $\left(\mathrm{S}_{\mathrm{yx}} \%\right)$ (DRAPER; SMITH, 1998), calculados para a variável probabilidade de mortalidade e também para a estimativa do número de árvores mortas por hectare. Ainda, foram calculadas as estatísticas viés ( vies $=\sum_{i=1}^{n} \frac{y_{i}-\hat{y}_{i}}{n}$ ), viés absoluto (vies absoluto $=\sum_{i=1}^{n} \frac{\left|y_{i}-\hat{y}_{i}\right|}{n}$ ) (MÔRA, 2015; MURTA JÚNIOR et al., 2015) e critério de informação de Akaike (CIA) (FLORIANO et al., 2006). Finalmente, a dispersão residual foi verificada para os dois modelos mais adequados em estimar a mortalidade por hectare.

\section{RESULTADOS E DISCUSSÃO}

\section{Análise da mortalidade do povoamento}

$\mathrm{O}$ número de árvores mortas por classe de sítio e idade está apresentado na Figura 1. Houve aumento da mortalidade de árvores com o decorrer da idade, sendo mais evidente nos sítios I e II, assumindo uma tendência similar àquela descrita em Schneider et al. (2015). De acordo com Zhao et al. (2007), a probabilidade de sobrevivência diminui com o aumento da idade do povoamento e com a classe de sítio. Em geral, o número de árvores mortas por idade foi maior para o sítio I, evidenciando a existência de maior competição no sítio mais produtivo, com início antecipado em relação às demais classes de sítio, dada à maior taxa de incremento das variáveis dendrométricas.

Além da irregularidade da ocorrência da mortalidade, oscilações ocorreram devido às mesmas parcelas não serem remedidas ao longo de todo o ciclo do povoamento. Colunas ausentes representam idades as quais não foram representadas na base de dados desta pesquisa, em que somente a classe de sítio II foi representada em todas as idades. Do total de parcelas empregado neste estudo, em $57 \%$ houve casos de mortalidade regular. 

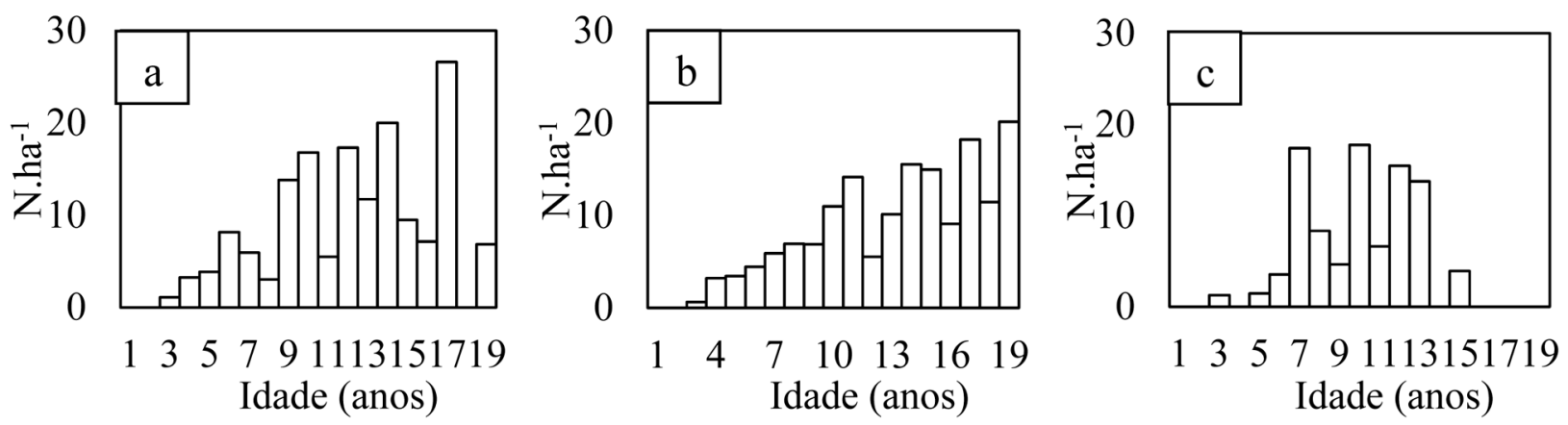

Figura 1. Número de árvores mortas por hectare e por idade, para sítio I (a), sítio II (b) e sítio III (c).

Figure 1. Number of dead trees per hectare and age, for site I (a), site II (b) and site III (c).

Na Figura 2 está apresentada a dinâmica do número de árvores vivas e mortas do povoamento, para as idades de 5, 10, 15 e 20 anos. Observa-se o avanço da mortalidade nas classes diamétricas com o decorrer da idade. Na idade de 5 anos, em média, houve poucos casos de mortalidade, mas a partir dos 10 anos, ocorrências ficaram mais evidentes. A mortalidade ocorreu somente nas classes iniciais, como esperado, por se tratarem de árvores dominadas ou suprimidas, que sofrem maiores efeitos da competição.

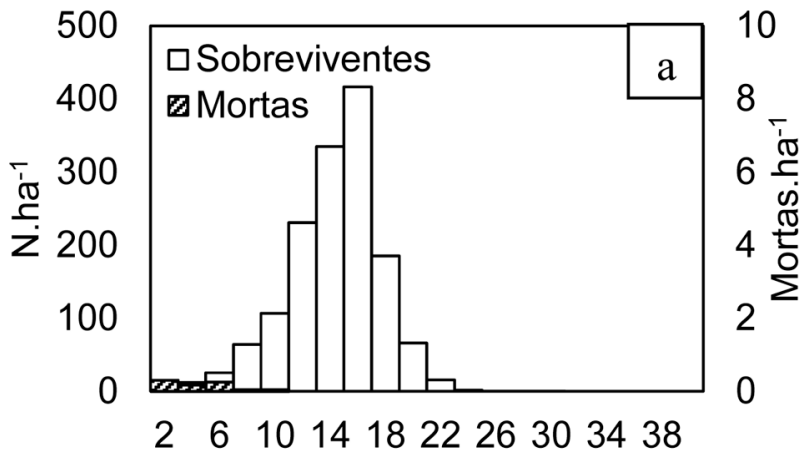

Centro da classse de $\mathrm{d}(\mathrm{cm})$

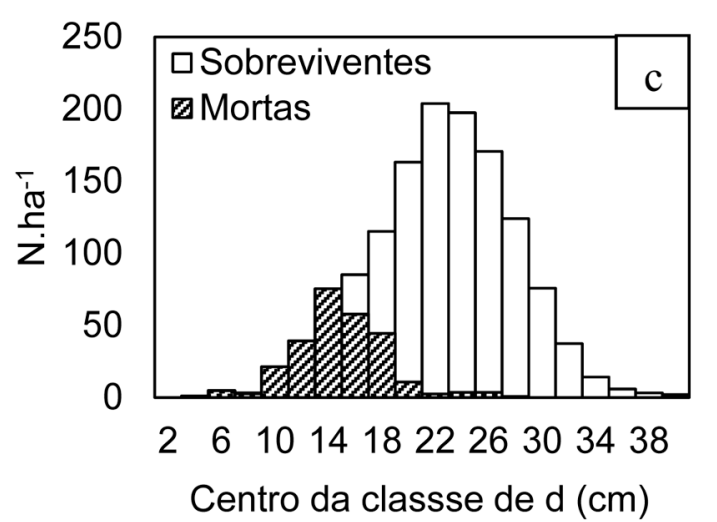

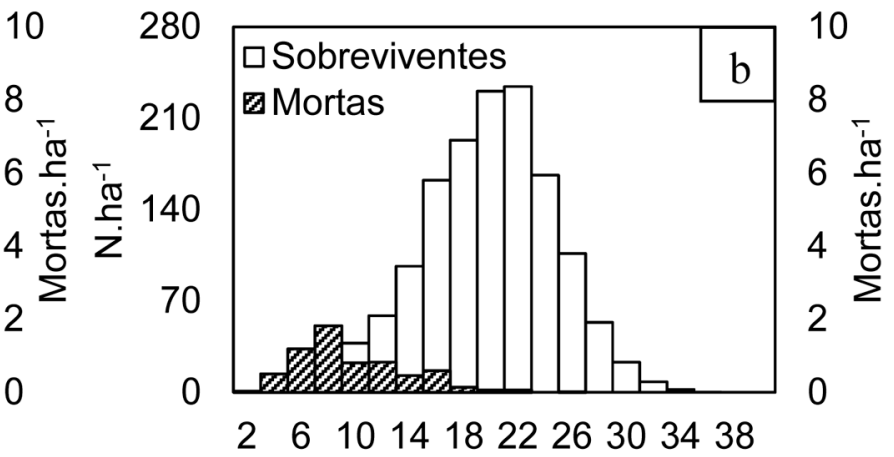

Centro da classse de $\mathrm{d}(\mathrm{cm})$

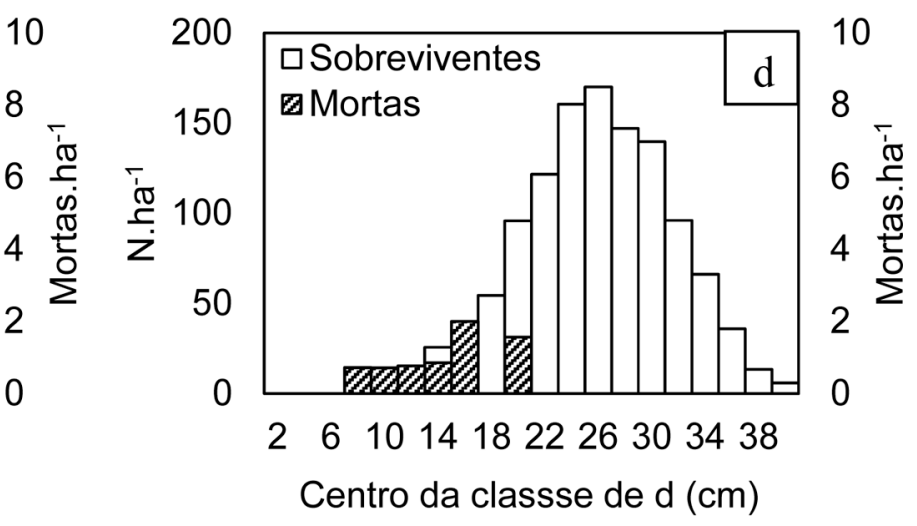

Figura 2. Dinâmica do número de árvores vivas e mortas por hectare e por classe de diâmetro, para as idades de 5 anos (a), 10 anos (b), 15 anos (c) e 20 anos (d).

Figure 2. Dynamic of the number of alive and dead trees per hectare and diameter class, for the ages of 5 years (a), 10 years (b), 15 years (c) and 20 years (d).

Por meio de análises nos dados, pôde ser verificado que a mortalidade ocorreu até a classe de $28 \mathrm{~cm}$, sendo que, após essa classe, não foram constatadas ocorrências de mortalidade. Como forma de determinar a região de ocorrência da mortalidade e, consequentemente, dos limites de aplicação da função de mortalidade, foi analisada a relação entre o diâmetro máximo em que foi registrada mortalidade e o diâmetro médio aritmético da parcela. Em média, a área de ocorrência de mortalidade de árvores se situou entre o diâmetro mínimo e 75\% do diâmetro médio aritmético da parcela. O emprego de apenas um percentual para o povoamento se deu devido os resultados terem sido muito próximos nas diferentes classes de sítio. Essas análises foram essenciais para determinar as condições para aplicação das equações de probabilidade de mortalidade e, com isso, reduzir problemas em superestimar essa variável. 


\section{Modelos de mortalidade}

Na Tabela 3 estão apresentados os coeficientes estimados e estatísticas de avaliação para os modelos testados. Os coeficientes $\beta_{4}$ do modelo exponencial com os índices de competição IC.1 e IC. 2 foram não significativos ${ }^{\left({ }^{n}\right.}$ ), demonstrando que a presença da variável altura média da classe não contribuiu de maneira significativa para a estimativa da probabilidade de mortalidade. Todos os demais coeficientes foram significativos $\left({ }^{*}\right)$ a $5 \%$ de significância.

Em todos os casos, o $\mathrm{S}_{\mathrm{yx}} \%$ apresentou-se muito elevado, devido ao excesso de valores "zeros" nas observações, já que houve mais casos de não mortalidade do que a existência de mortalidade. Conforme o viés, ambos os modelos logísticos subestimaram a probabilidade de mortalidade. Porém, baseando-se nos valores absolutos dessa estatística, não houve discrepâncias entre os modelos testados. Finalmente, os valores do critério de informação de Akaike se apresentaram próximos perante os diferentes modelos, porém, sugerindo ligeira superioridade dos modelos exponencial e de Buchman. O melhor modelo exponencial e o melhor de Buchman foram considerados os mais adequados, empregando os índices IC.1 e IC.3, respectivamente.

Tabela 3. Coeficientes estimados e estatísticas de avaliação dos modelos de probabilidade de mortalidade. Table 3. Estimated coefficients and evaluation statistics of the probability of mortality models.

\begin{tabular}{|c|c|c|c|c|c|c|c|c|c|c|c|c|c|c|}
\hline Modelo & IC & $\beta_{0}$ & $\beta_{1}$ & $\beta_{2}$ & $\beta_{3}$ & $\beta_{4}$ & $\beta_{5}$ & $\beta_{6}$ & $\beta_{7}$ & Syx & $\begin{array}{c}\text { Syx } \\
(\mathrm{Pm}) \%\end{array}$ & Viés & $\begin{array}{l}\text { iés } \\
\text { os. }\end{array}$ & CIA \\
\hline \multirow{3}{*}{$\begin{array}{c}\text { (1) } \\
\text { Logístico (a) }\end{array}$} & & & & - & - & 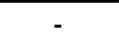 & 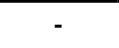 & 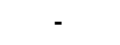 & & & & & 0,02 & \\
\hline & IC. 2 & $6,9639^{*}$ & ,2229* & - & - & - & - & 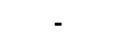 & 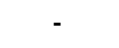 & 06 & 426,5 & & 0,02 & .811 \\
\hline & IC. 3 & $2,1082^{*}$ & $4,2328^{*}$ & - & - & - & - & - & 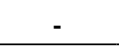 & 0,06 & 449,8 & & 0,02 & -78.822 \\
\hline \multirow{3}{*}{$\begin{array}{c}\text { (2) } \\
\text { Logístico (b) }\end{array}$} & IC.1 & & $6131^{*}$ & 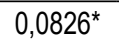 & 235 & 201 & 14 & & 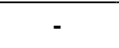 & & & & 0,02 & \\
\hline & IC. 2 & $-6,4157^{*}$ & $-0,0386^{*}$ & $-0,0500^{*}$ & $0,0434^{*}$ & $0,2600^{*}$ & & & - & 0,05 & 394,2 & & 0,02 & -78.568 \\
\hline & IC. 3 & $-5,0310^{*}$ & $-5,6926^{*}$ & $0,1197^{*}$ & $0,0726^{*}$ & $33^{*}$ & & 0,0 & - & 0,05 & 392,0 & & 0,02 & -78.711 \\
\hline \multirow{3}{*}{$\begin{array}{c}(3) \\
\text { Exponencial }\end{array}$} & IC.1 & & & & & & & & & & & &, 02 & \\
\hline & IC. 2 & $-0,0043^{*}$ & $-6,0870^{*}$ & $-0,01$ & $-0,0262^{*}$ & $0,0163^{\text {ns }}$ & $1^{*}$ & $-0,7$ & & 0,05 & 397,5 & & 0,02 & -78.417 \\
\hline & IC. 3 & $-0,0033^{*}$ & $-4,8393^{*}$ & $-4,6642^{*}$ & $0,1080^{*}$ & $0,0408^{*}$ & $59^{*}$ & $-0,8$ & $26^{*}$ & 0,05 & 396,3 & 00 & 0,02 & -78.495 \\
\hline \multirow{3}{*}{$\begin{array}{c}(4) \\
\text { Buchman }\end{array}$} & IC.1 & $-0,0020^{*}$ & $5,9887^{\star}$ & & & & & & & 0,05 & 39 & & 0,02 & -78.637 \\
\hline & IC. 2 & $-0,0031^{*}$ & $6,2575^{\star}$ & $0,0585^{\star}$ & $-0,2574^{*}$ & $0,0524^{*}$ & $0,9950^{*}$ & $-0,0518^{*}$ & $-0,0408^{*}$ & 0,05 & 393,8 & & 0,02 & -78.724 \\
\hline & IC. 3 & $-0,0021^{*}$ & $5,0000^{*}$ & $5,3272^{*}$ & $-0,1623^{*}$ & $-0,1095^{*}$ & $1,1327^{*}$ & $-0,0295^{*}$ & $-0,0677^{*}$ & 0,05 & 1391,8 & 0,0000 & 0,02 & -78.595 \\
\hline
\end{tabular}

Em que: ICi = índice de competição, em que IC.I corresponde ao BAI ( $\mathrm{d}_{i}^{2} \mathrm{q}^{-2}$ ), IC.2 refere-se ao BAL $\left(\sum_{i=1} \mathrm{~g}_{\mathrm{i}}\right)$, e IC.3 representa o índice de Tomé e Burkhart (1989) $\left(\mathrm{d}_{1} \mathrm{~d}_{\max }^{-1}\right)$; Syx\% = erro padrão da estimativa percentual; Viés abs. = viés absoluto; CIA = critério de informação de Akaike; $\beta_{\mathrm{i}}$ = coeficientes estimados; * e ns = coeficiente significativo e não significativo, respectivamente, a $5 \%$ de significância estatística.

Calculando o erro padrão da estimativa para o número de árvores mortas por hectare $\left(\mathrm{S}_{\mathrm{yx} \text { (N.mortas) }}\right)$, tanto para o modelo exponencial (IC.1) quanto para o de Buchman (IC.7), os valores foram inferiores àqueles da Tabela 3, sendo de 128,4\% e 159,7\%, respectivamente.

Na Figura 3 pode ser observada a dispersão dos resíduos para as estimativas do número de árvores mortas por hectare, em função da idade, para o modelo exponencial (a) e de Buchman (b). Ambos os modelos apresentaram tendência semelhante, os quais superestimaram o número de árvores mortas por hectare, principalmente em idades mais avançadas, e em maior intensidade para o modelo de Buchman.

Pelas análises anteriores, nenhum dos modelos testados apresentou superioridade evidente sobre o outro. Dessa forma, ambos os modelos foram empregados para estimar o número de árvores mortas por hectare, para cada classe de sítio, como pode ser observado na Figura 4. Para o sítio I, houve tendência de subestimação do número de árvores mortas nas idades iniciais, alterando para superestimação a partir dos 17 anos, para o ambos os modelos. Para a classe de sítio II, ocorreu tendência de superestimação, para os dois modelos testados. Finalmente, dada à uma distribuição mais irregular da mortalidade no sítio III, as estimativas não apresentaram uma tendência definida, entretanto, demonstraram comportamento similar às observações.

Conforme Eid e Tuhus (2001), o maior desafio dos modelos de mortalidade está em estimar satisfatoriamente sua probabilidade, sobretudo nos estágios iniciais e finais de crescimento. De acordo com Martins (2011), isso é resultado da falta de uma tendência característica da mortalidade em função da idade. 

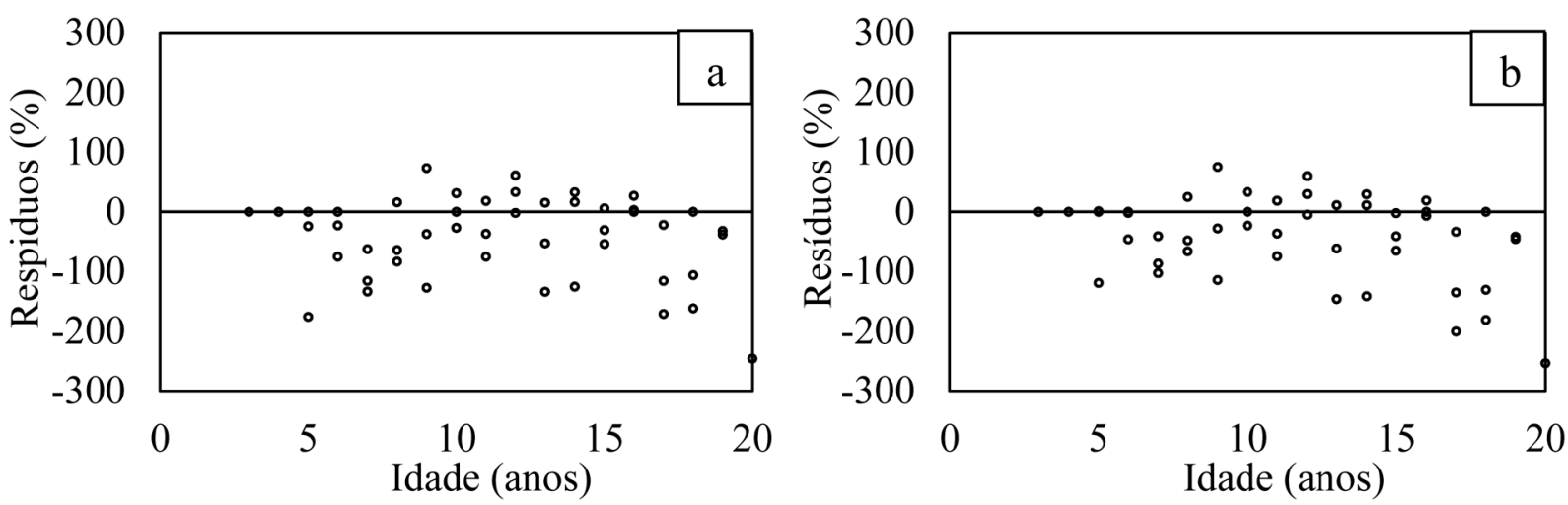

Figura 3. Dispersão residual para a estimativa de árvores mortas por hectare em função da idade, para o modelo exponencial (a) e de Buchman (b).

Figure 3. Residual dispersion to estimate dead trees per hectare in function of the age, for exponential (a) and Buchman (b) models.

Modelo Exponencial

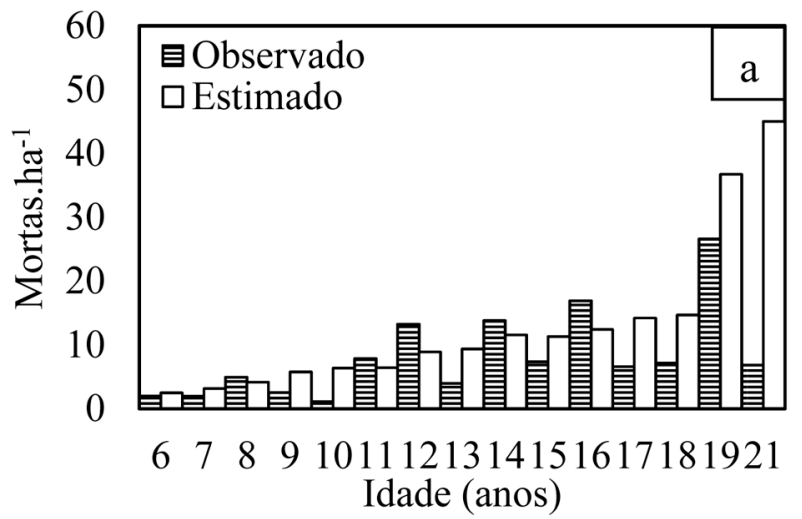

Modelo de Buchman
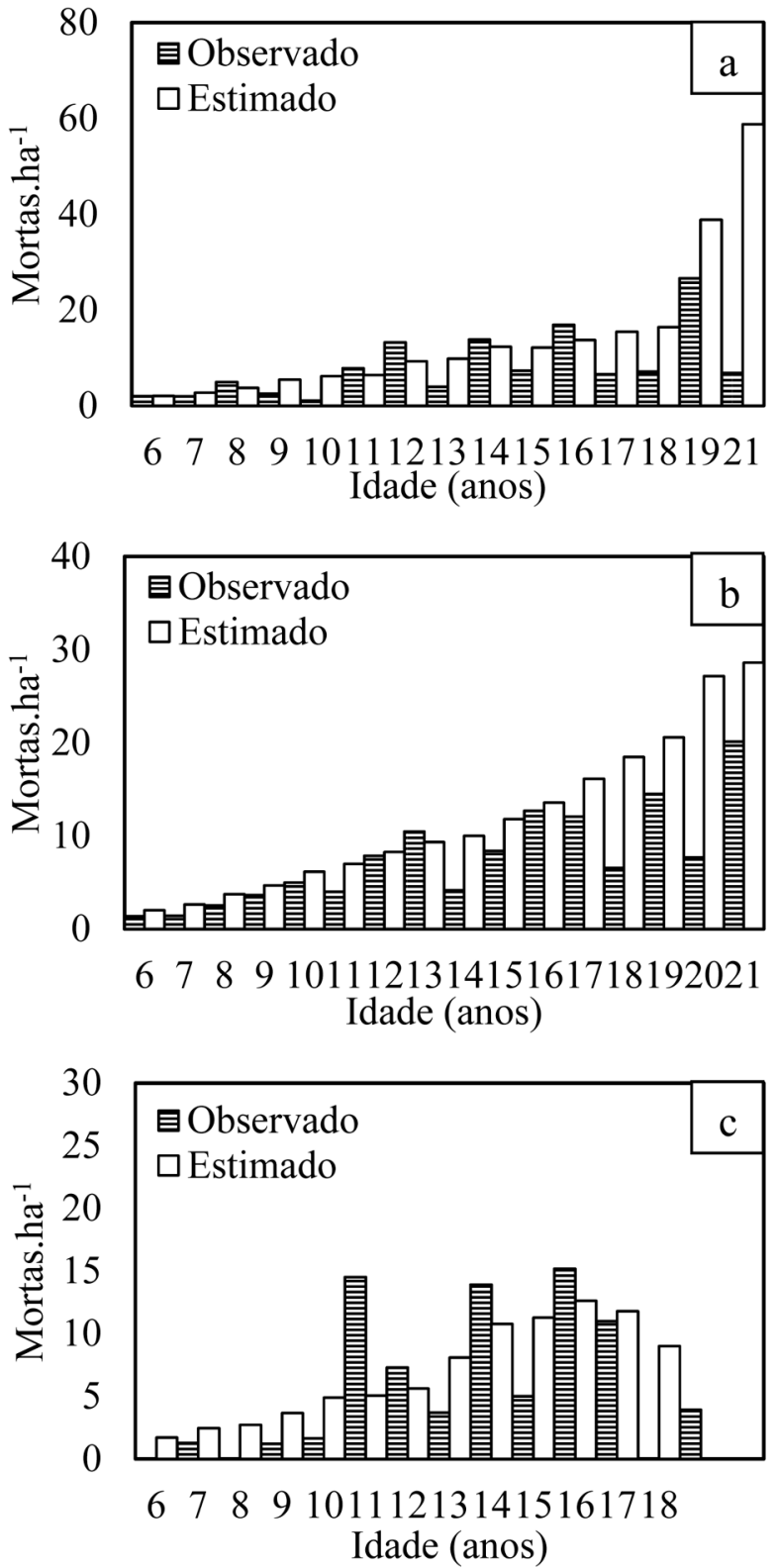

Figura 4. Valores observados e estimados do número de árvores mortas por hectare, para o modelo exponencial e de Buchman, para o sítio I (a), sítio II (b) e sítio III (c).

Figure 4. Observed and estimated values of the number of dead trees per hectare, for exponential and Buchman model for site I (a), site II (b) and site III (c). 
Miranda et al. - Modelagem da mortalidade em povoamentos de Pinus taeda L.

Diante dos resultados, o modelo exponencial, empregando o índice de competição IC.1 (BAI), foi considerado o mais adequado em estimar a mortalidade regular do povoamento em estudo. Isso porque, embora os valores obtidos pelos dois modelos em avaliação se apresentaram similares (Figura 4), o número de árvores mortas por hectare estimado pelo modelo de Buchman se distanciou mais dos respectivos valores observados, principalmente para a classe de sítio I, onde ocorreram superestimativas.

O índice de Stage (1973) (BAI) também foi empregado em outros trabalhos na avaliação da mortalidade. Como exemplo, Glover e Hool (1979) empregaram o índice BAI no desenvolvimento de um modelo geral de mortalidade de árvores individuais de P. taeda, com base na função Weibull, utilizando características das árvores e do povoamento. Os dados foram provenientes de um experimento de adubação nitrogenada, situado no estado americano de Alabama. Esses autores concluíram que o modelo estimou satisfatoriamente a mortalidade do povoamento.

West (1981) desenvolveu uma função de mortalidade baseada naquela proposta por Glover e Hool (1979), empregando também o índice BAI para espécies de eucaliptos, com idade de 14 a 83 anos. Os dados foram obtidos em uma área localizada na Tasmânia, Austrália. Esse autor concluiu que o modelo foi adequado e gerou estimativas precisas da distribuição diamétrica dos povoamentos.

\section{CONCLUSÕES}

A mortalidade de árvores se situou nas classes inferiores de diâmetro, de modo que a área de ocorrência da mortalidade regular, baseada na relação entre o diâmetro máximo de ocorrência de mortalidade com o diâmetro médio aritmético da parcela, foi útil para determinar as condições para aplicação da função de mortalidade.

O modelo exponencial foi aquele que melhor estimou o número de árvores mortas por hectare, para as três classes de sítio.

\section{REFERÊNCIAS BIBLIOGRÁFICAS}

ASSMANN, E. The principles of forest yield study: studies in the organic production, structure, increment and yield of forest stands. Oxford: Pergamon Press. 1970. 506 p.

BUCHMAN, R. G.; PEDERSON, S. P.; WALTERS, N. R. A tree survival model with application to species of the Great Lakes region. Canadian Journal of Forest Research, Ottawa, v.13, n.4, p.601-608, 1983.

BUFORD, M. A.; HAFLEY, W. L. Probability distributions as models for mortality. Forest Science, Bethesda, v.31, n.2, p.331-341, 1985.

CAO, Q. V. A Method to distribute mortality in diameter distribution models. Forest Science, Bethesda, v.43, n.3, p.435-442, 1997.

CASTEL-BRANCO, D. A. A. S. Análise da mortalidade em plantações jovens de sobreiro (Quercus suber L.) e sua relação com a qualidade da estação. 2014, 68 p. Dissertação (Mestrado em Engenharia Florestal e dos Recursos Naturais) - Instituto Superior de Agronomia, Lisboa, 2014.

CASTRO, R. V. O. Modelagem de árvore individual para uma floresta estacional Semidecidual utilizando redes neurais. 2012. 105 p. Tese (Doutorado em Ciência Florestal) - Universidade Federal de Viçosa, Viçosa, 2012.

CASTRO, R. V. O. Modelagem do crescimento em nível de árvores individuais utilizando redes neurais e autômatos celulares. 2011. 80 p. Dissertação (Mestrado em Ciência Florestal) - Universidade Federal de Viçosa, Viçosa, 2011.

DOBBERTIN, M.; BIGING, G. S. Using the non-parametric classifier CART to model forest tree mortality. Forest Science, Bethesda, v. 44, n. 4, p. 507-516, 1998. 
DRAPER, N. R.; SMITH, H. Applied regression analysis. 3. ed. New York: John Wiley \& Sons, 1998. 706 p.

EID, T.; TUHUS, E. Models for individual tree mortality in Norway. Forest Ecology and Management, Amsterdam, v. 154, n. 1-2, p. 69-84, 2001.

FIGURA, M. A. A distribuição de Weibull na descrição da estrutura diamétrica de Eucalyptus grandis: Um enfoque sobre o método dos momentos. 2010, 98 p. Dissertação (Mestrado em Engenharia Florestal) Universidade Federal do Paraná, Curitiba, 2010.

FLEWELLING, J. W.; MONSERUD, R. A. Comparing methods for modelling tree mortality. In CROOKSTON, N. L., HAVIS, R. N. (Eds.). Second Forest Vegetation Simulator Conference, Fort Collins: USDA Forest Service, p.168-177. 2002.

FLORIANO, E. P.; MÜLLER, I.; FINGER, C. A. G.; SCHNEIDER, P. R. Ajuste e seleção de modelos tradicionais para série temporal de dados de altura de árvores. Ciência Florestal, Santa Maria, v. 16, n. 2, p. 177-199, 2006.

GLOVER, G. R.; HOOL, J. N. A basal area ratio predictor of loblolly pine plantation mortality. Forest Science, Bethesda, v. 25, n. 2, p. 275-282, 1979.

LIEBSCH, D.; MIKICH, S. B.; OLIVEIRA, E. B.; MOREIRA, J. M. M. A. P. Descascamento de Pinus taeda por macacos-prego (Sapajus nigritus): tipos e intensidades de danos e seus impactos sobre o crescimento das árvores. Scientia Forestalis, Piracicaba, v.43, n.105, p.37-49, 2015.

MABVURIRA, D.; MIINA, J. Individual-tree growth and mortality models for Eucalyptus grandis (Hill) Maiden plantations in Zimbabwe. Forest Ecology and Management, Amsterdam, v. 161, n. 1-3, p. 231-245, 2002.

MACHADO, S. A.; TONON, A. E. N.; FIGUEIREDO FILHO, A.; OLIVEIRA, E. B. Comportamento da mortalidade natural em bracatingais nativos em diferentes densidades iniciais e classes de sítio. Ciência Florestal, Santa Maria, v.12, n.2, p.41-50, 2002.

MARTINS, F. B. Modelagem de crescimento em nível de árvore individual para plantios comerciais de eucaliptos. 2011. 143 p. Tese (Doutorado em Ciência Florestal) - Universidade Federal de Viçosa, Viçosa, 2011.

MARTINS, F. B.; SOARES, C. P. B.; LEITE, H. G.; SOUZA, A. L.; CASTRO, R. V. O. Índices de competição em árvores individuais de eucalipto. Pesquisa Agropecuária Brasileira, v.46, n.9, p.1.089-1.098, 2011.

MIRANDA, R. C.; CAMPOS, J. C. C.; PAULA NETO, F.; OLIVEIRA, L. M. Predição da mortalidade regular para eucalipto. Revista Árvore, Viçosa, v. 13, n. 2, p. 152-173, 1989.

MÔRA, R. Funções de afilamento de forma variável e modelagem de efeitos mistos em fustes de Pinus taeda e Eucalyptus saligna. 2015, 277 p. Tese (Doutorado em Engenharia Florestal) - Universidade Federal do Paraná, Curitiba, 2015.

MURTA JÚNIOR, L. S.; OLIVEIRA, M. R. L.; NOGUEIRA, G. S.; LEITE, H. G.; CASTRO, R. V. O.; ABRAHÃO, C. P. Avaliação do método da similaridade de perfis na estimação do volume de árvores. Scientia Forestalis, Piracicaba, v. 43, n. 106, p. 435-444, 2015.

NUNES, J. R. S.; FIER, I. S. N.; SOARES, R. V.; BATISTA, A. C. Desempenho da fórmula de Monte Alegre (FMA) e da fórmula de Monte Alegre Alterada (FMA+) no distrito florestal de Monte Alegre. Floresta, Curitiba, v.40, n.2, p.319-326, 2010.

PAYANDEH, B. Some applications of nonlinear regression models in forest research. The Forestry Chronicle, Quebec, v. 59, n. 5, p.244-248, 1983. 
Miranda et al. - Modelagem da mortalidade em povoamentos de Pinus taeda L.

SCHNEIDER, P. R.; FINGER, C. A. G.; SCHNEIDER, P. S. P.; FLEIG, F. D.; CUNHA, T. A. Influência do espaçamento no autodesbaste de povoamento monoclonal de Eucalyptus saligna Smith. Ciência Florestal, Santa Maria, v.25, n.1, p.119-126, 2015.

SIMS, A.; KIVISTE, A.; HORDO, M.; LAARMANN, D.; GADOW, K. von Estimating tree survival: a study based on the Estonian Forest Research Plots Network. Annales Botanici Fennici, v.46, n.4, p.336-352, 2009.

STAGE, A. R. Prognosis model for stand development. Colorado: USDA Forest Service, 1973. (Research Paper INT-137).

TECK, R. M.; HILT, D. E. Individual-tree probability of survival model for the Northeastern United States. Radnor: U.S. Department of Agriculture, 1990. 10 p. (Research Paper. NE-642)

THAPA, R. Modeling Mortality of Loblolly Pine (Pinus taeda L.) Plantations. 2014. 187 p. (Phd. Thesis Philosophy in Forestry) - Faculty of the Virginia Polytechnic Institute and State University, Virginia, 2014.

TOMÉ, M.; BURKHART, H. E. Distance-dependent competition measures for predicting growth of individual trees. Forest Science, Bethesda, v.35, n.3, p.816-831, 1989.

WEISKITTEL, A. R.; HANN, D. W.; KERSHAW, J. A. J; VANCLAY, J. K. Forest Growth and Yield Modeling. Oxford: John Wiley \& Sons, 2011. 415 p. 2011.

WEST, P. W. Simulation of diameter growth and mortality in regrowth eucalypt forest of southern Tasmania. Forest Science, Bethesda, v. 27, n. 3, p. 603-616, 1981.

YANG, Y.; TITUS, S. J.; HUANG, S. Modeling individual tree mortality for white spruce in Alberta. Ecological Modelling, Kidlington, v. 163, n.3, p. 209-222, 2003.

ZHAO D.; BORDERS, B.; WANG, M.; KANE, M. Modeling mortality of second-rotation loblolly pine plantations in the Piedmont/Upper Coastal Plain and Lower Coastal Plain of the southern United States. Forest Ecology and Management, Amsterdam, v. 252, n.1-3, p. 132-143, 2007.

Recebido em 03/04/2016

Aceito para publicação em 30/03/2017 